\title{
The efficacy of lamotrigine after failure of the first administration of valproate in treating epilepsy: a systematic review and meta- analysis
}

\author{
Jia He ${ }^{1}$, Xueying $\mathrm{Wu}^{1}$, Dong Zhou ${ }^{2}$ \\ ${ }^{1}$ Department of Neurology, 363 Hospital, Chengdu, China; ${ }^{2}$ Department of Neurology, West China Hospital, Sichuan University, Chengdu, China \\ Contributions: (I) Conception and design: J He, X Wu; (II) Administrative support: D Zhou; (III) Provision of study materials or patients: All authors; \\ (IV) Collection and assembly of data: All authors; (V) Data analysis and interpretation: All authors; (VI) Manuscript writing: All authors; (VII) Final \\ approval of manuscript: All authors. \\ Correspondence to: Dong Zhou. Department of Neurology, West China Hospital, Sichuan University, No. 37 Guoxue Alley, Wuhou District, \\ Chengdu, China. Email: zhoudong66@yahoo.de.
}

Backgroundk Epilepsy is a long-term recurrent chronic brain disease that can cause significant emotional burden to the patient and their family, as well as huge economic costs to society. Timely and accurate diagnosis of epilepsy, together with early and standardized treatments can effectively control seizures and restore the patient's quality of life and reduce the economic burden. This meta-analysis examined the efficacy of lamotrigine administration in patients with epilepsy.

Methods: A literature search was performed in the PubMed, Embase, and OVID-Medline databases to identify articles related to epilepsy and lamotrigine that were published from the establishment of the database to April 2021. The keywords used for the literature search included "epilepsy", "sodium valproate", "lamotrigine", "effectiveness", and "therapeutic effect". It uses Cochrane review manual 5.3 to evaluate the quality of the included literature and review manager 5.3 software for meta-analysis.

Results: A total of 9 studies involving 1,864 patients with epilepsy were included in this meta-analysis. The results revealed that, after treatment failure with the first drug of valproic acid, the total effective rate of lamotrigine treatment had an odds ratio (OR) of 2.21 with a $95 \%$ confidence interval (CI) of 1.15 to 4.27 $(\mathrm{Z}=2.37 ; \mathrm{P}=0.02)$. The total adverse reaction rate $(\mathrm{OR}=0.70 ; 95 \% \mathrm{CI}: 0.55$ to $0.88 ; \mathrm{Z}=2.98 ; \mathrm{P}=0.003)$ and the improvement rate of epilepsy associated with lamotrigine treatment (OR =4.22; 95\% CI: 1.00 to 17.84; $\mathrm{Z}=1.96 ; \mathrm{P}=0.05$ ) were all significantly higher than that of other drug treatments.

Discussion: A total of 9 articles were included in this meta-analysis to examine the efficacy of lamotrigine in the treatment of epilepsy. The clinical efficacy of lamotrigine addition therapy was found to be superior to lamotrigine replacement therapy, and the incidence of adverse reactions was lower than that of lamotrigine replacement therapy. However, due to the low methodological quality of the included literatures, this conclusion should be further verified using large sample and high-quality randomized double-blinded experiments.

Keywords: Epilepsy; sodium valproate; lamotrigine; meta-analysis; effectiveness

Submitted Nov 08, 2021. Accepted for publication Dec 24, 2021.

doi: 10.21037/apm-21-3555

View this article at: https://dx.doi.org/10.21037/apm-21-3555 


\section{Introduction}

Epilepsy is a chronic brain disorder characterized by sudden, recurrent, and transient central nervous system dysfunction caused by the excessive firing of neurons in the brain (1). The clinical manifestations include disorders of movement, sensation, consciousness, behavior, and autonomic nerve function (2), depending on the site of the invaded neurons and the range of discharge diffusion. Epilepsy is one of the top 10 medical problems worldwide, with an annual incidence of 50-70/100,000 and a prevalence of about $5 \%$. The mortality rate is $1.3-3.6$ per 100,000 , which is 2-3 times that of the general population. At present, it is the third most common disease in neurology after cerebrovascular disease and dementia $(3,4)$. Unfortunately, treatment is ineffective for a considerable number of patients, leading to serious physical and mental stress for the patients and their families, as well as significant strain on the health system (5).

While the etiology of epilepsy remains unclear, studies have suggested that it may involve cortical dysplasia, brain tumors, head injuries, and cerebrovascular diseases $(6,7)$. In addition, central nervous system infections, parasites, and genetic metabolic diseases may also lead to epilepsy (8). While pathogenesis of epilepsy is unclear, possible mechanisms involving ion channels and abnormal brain neural networks have been suggested $(9,10)$.

Clinical seizures occur when multiple neurons in the brain fire abnormally at the same time. When the abnormal discharge of a neuron enters the local neural network and propagates, the abnormal current can be increased or decreased by the gain or inhibition of the excited or inhibitory neurons in the network (11). The form of seizures depends largely on where the abnormal firing of the neuron occurs in the brain (12). Different discharge sites determine different seizure types in the clinical manifestations of epilepsy. Abnormal discharge in focal epilepsy is mostly confined to a certain brain region (13). When the epileptic discharge of neurons in the brain is widespread, reaching the large bilateral brain, it leads to the seizure of comprehensive epilepsy (14). Partial epileptic complex seizures are caused by the abnormal firing of neurons that diffuse to the limbic system, and absence seizures are caused by the suppression of abnormal electrical waves transmitted to the thalamic neurons (15). The clinical diagnosis of epilepsy should first determine whether the clinical manifestations of the patient satisfies the characteristics of clinical seizures.
The basic characteristics of epileptic seizure in humans are epileptic discharge on electroencephalogram (EEG) and clinical seizures, and the main basis of diagnosis is the history of the epileptic patient (16). Subsequently, the type epileptic seizure should be identified through the clinical manifestations of the patient, so as to clarify whether it is an epileptic syndrome (17). Different types of epileptic seizures have different pathophysiological mechanisms and anatomical basis, and the appropriate therapeutic drugs should be used. Epilepsy syndrome is composed of a group of symptoms and signs, is a specific epilepsy phenomenon, which includes not only the type of seizures, but also a special cause, pathological mechanism, prognosis, and outcome, and is differs from general seizures in terms of drug selection and treatment (18). In addition to primary epilepsy, the cause of secondary epilepsy should be clear, and computed tomography (CT), magnetic resonance, isotope scan, cerebrovascular angiography, and other auxiliary means can be considered for examination (19).

The ideal goal of epilepsy treatment is complete seizure control with no or minimal drug side effects and minimal impact on the patient's quality of life. Modern epilepsy treatment mainly includes etiology treatment, drug treatment, surgical treatment, and other physical therapy (20). Studies have shown that patients with no clear cause or those who have a cause but the cause itself cannot be eradicated should receive aggressive drug treatment (21). The choice of treatment drugs should be based on the different types of seizures. Carbamazepine is the first choice for the treatment of partial seizures, partial systemic seizures, and mandatory seizures, followed by phenytoin sodium, phenobarbital, and valproic acid $(22,23)$. Valproic acid is the first-choice treatment for systemic spasmodic seizures and spastic seizures, followed by carbamazepine, topiramate, and oxcarbazepine (24). The use of sodium valproate drug can cause blood cell reduction and liver function damage in patients, and long-term use may cause brain damage in patients (25). Lamotrigine is a new type of anti-epileptic drug, which is a voltage-based sodium ion channel blocker and can be used in the treatment of epilepsy. But clinically, lamotrigine can only be used in the treatment of children or adults over 12 years old. For these patients, surgical treatment should be performed as soon as possible. At present, the various surgical treatments performed in China for epilepsy include selective amygdalohippocampectomy, cerebral hemispherectomy, anterior temporal lobectomy, and resection of the epileptic focus outside the temporal lobe (26). 
At present, there are many observational studies on the clinical efficacy of lamotrigine or valproic acid in the treatment of epilepsy. However, after the first drug treatment of valproic acid, there are few studies on the effect of lamotrigine treatment. Therefore, this metaanalysis examined the effectiveness of lamotrigine after the failure of sodium valproate in the treatment of epilepsy. We present the following article in accordance with the PRISMA reporting checklist (available at https://apm. amegroups.com/article/view/10.21037/apm-21-3555/rc).

\section{Methods}

\section{Literature search strategy}

A comprehensive and systematic literature search was conducted according to the Cochrane Handbook of Systematic Reviews of Interventions, and meta-analysis was performed following the PRISMA. The PubMed, Embase, and OVID-Medline database was searched for scientific conference literature and established articles related to patients treated for epilepsy. The keywords and medical titles used in specific searches included the following: "valproate", "lamotrigine", "epilepsy", and "effectiveness". The incidence rate of epilepsy was included in the study as an indirect method to adjust the scope, course, and region of the disease.

\section{Inclusion and exclusion criteria for the articles}

Articles were included in the meta-analysis if they satisfied the following inclusion criteria: (I) the literatures were all randomized controlled trials (RCTs); (II) the subjects in the study were patients with epilepsy and the type of epilepsy was diagnosed by EEG and clinical examination according to the criteria established by the International League Against Epilepsy (ILAE); (III) the patients were non-responsive to valproate therapy; (IV) the course of the disease was monitored for 3 months or more, and the patients had good medication compliance and follow-up could be completed; (V) patients in the treatment group were administered lamotrigine and lamotrigine replacement therapy was used in the control group; (VI) the evaluation indicators included total effective rate, total incidence of adverse reactions, and the incidence of adverse reactions in each system; and (VII) no experimental data was missing, the sample size was exact, and the intergroup data of the study object was complete.

The following exclusion criteria were applied: (I) duplicate publications, including multiple articles reporting the same clinical trial using different evaluation indicators, in which case, the first published data were extracted and included in this study; (II) literature that is inconsistent with the evaluation indexes of this study or did not provide valid data; (III) low-quality literature; and (IV) literature that is repeated and reprinted.

\section{Literature screening}

The two researchers independently screened the titles and abstracts according to the selection criteria, and relevant data were extracted and the quality of the literature was evaluated. Any disagreements were resolved via discussion or consultation with a third researcher. The full text articles were then retrieved for data extraction. Note express 2.0 was used for literature management and duplicate literature was deleted. The inclusion of literature was checked according to the inclusion and exclusion criteria mentioned above and the relevant literature were traced.

\section{Data extraction}

The two researchers independently extracted the relevant information from all eligible studies using a predefined data extraction table including author, year of publication, sample size, age, country, gender, degree of disease, and course of disease. Under outcome indicators: total treatment effect, incidence of adverse events, and improvement rate of epilepsy, a total of three indicators were used to evaluate the effect of epilepsy treatment. In the event of missing data, the original authors of the literature were contacted via email. If the requested data was not available, the Cochrane Evaluation Manual was used for relevant transformation, such as the calculation of standard deviation of continuous data.

\section{Quality assessment}

The quality of the included literature was assessed in accordance with the "risk of bias assessment" recommended in version 5.3 of the Cochrane System Review Manual. The evaluation includes the following seven items: (I) random grouping method; (II) allocate method; (III) blinding method between patients and researchers; (IV) effect of the blinding method; (V) integrity of the results; (VI) credibility of survey results; and (VII) other bias.

Is there a result the integrity of the credibility of 7 other 
6 survey bias about the item 7 of the RCT, "meet" said bias is small "not satisfied" refers to height bias, study is not fully detailed report, if no mention, danger is unknown evaluation including random sequence generation, allocation concealment, blinded.

Literature with a score of $1-3$ in the 4 dimensions of tracking/exit is considered low quality, and literature with a score of 4-7 is considered high quality.

\section{Data analysis and result display}

The forest plot clearly shows the results of individual studies and combines the corresponding confidence intervals (CIs) from the studies. When $\mathrm{I}^{2}<50 \%$ and $\mathrm{P}>0.05$, there was no statistical heterogeneity among the trials, and the fixed effects model was selected for meta-analysis. When $\mathrm{I}^{2} \geq 50 \%$ and $\mathrm{P} \leq 0.05$, statistical heterogeneity was present among the studies, and the random effects model was selected for meta-analysis. The combined effect size of the two groups of evaluation index data was used to determine the odds ratio $(\mathrm{OR})$ value and its $95 \% \mathrm{CI}$.

Results with high heterogeneity were analyzed with the article by article elimination method to explore the possible sources of heterogeneity, and sensitivity analysis was performed. Generally speaking, a particular study will have an impact on the comprehensive study under the following two circumstances: (I) if a study is deleted and the presumption of the size of the combined effect is $95 \%$ of the size of the combined effect; and (II) when a study is deleted, the results are significantly different. If one study affects the overall results with little difference, it indicates the sensitivity of the combined results and the results obtained are not stable. If the number of RCTs of an indicator is greater than or equal to 5 , publication bias was evaluated using the funnel plot.

\section{Statistical analysis}

The Review Manager 5.3 software (provided by the Cochrane Collaboration) was used for data processing and a $\mathrm{P}$ value $\leq 0.05$ was considered statistically significant.

\section{Results}

\section{Literature search results}

A total of 1,567 related documents were retrieved from the database and 313 articles that did not satisfy the inclusion and exclusion criteria were excluded. A brief review of the abstract was performed and 1,152 documents were excluded. The main text of the remaining 102 articles were reviewed and 89 documents were excluded. A total of 13 articles were reviewed in detail and 3 articles with unclear outcome indicators and 1 article with unclear groupings were excluded. Finally, a total of 9 articles (27-35) were included for meta-analysis. The basic process of the document retrieval and exclusion process is shown in Figure 1. The basic characteristics of the included literature are also detailed in Table 1.

\section{Bias-risk assessment of included articles}

The Cochrane Handbook (version 5.0.2) of the systematic review writing manual was used to evaluate the risk of bias in the 9 articles included in this study. Review Manager 5.3 was employed to output the risk of bias chart (Figures 2,3).

The Jadad scale was used to evaluate the quality of each included literature. All the 9 literatures included in the study were found to have a low risk of bias and therefore, satisfied the requirements of subsequent analyses (Table 2).

\section{Total effective rate}

A total of 9 literatures reported the total effective rate $\left(\mathrm{P}<0.00001 ; \mathrm{I}^{2}=83 \%\right)$ and the data was analyzed using the random effects model. Meta-analysis revealed that the total effective rate of the experimental group was significantly higher than that of the control group (OR $=2.21 ; 95 \%$ CI: 1.15 to $4.27 ; \mathrm{Z}=2.37 ; \mathrm{P}=0.02 ;$ Figure 4 ).

Taking the total effective rate as the index, the results of the inverted funnel plot showed that the scatter points of the research object are roughly funnel-like with the bottom facing down. On the horizontal axis, they are arranged roughly symmetrically, suggesting that there was no obvious bias (Figure 5).

\section{Total adverse reaction rate}

A total of 8 literatures reported the total adverse reaction rate $\left(\mathrm{P}=0.92 ; \mathrm{I}^{2}=0 \%\right)$ and the data was analyzed using the fixed effects model. Meta-analysis showed that the total adverse reaction rate in the experimental group was significantly lower than that in the control group (OR $=0.70 ; 95 \%$ CI: 0.55 to $0.88 ; Z=2.98 ; P=0.003 ;$ Figure 6).

The adverse reactions of the nervous system were used as indicators to draw an inverted funnel plot. The results 


\section{Identification of studies via databases and registers}
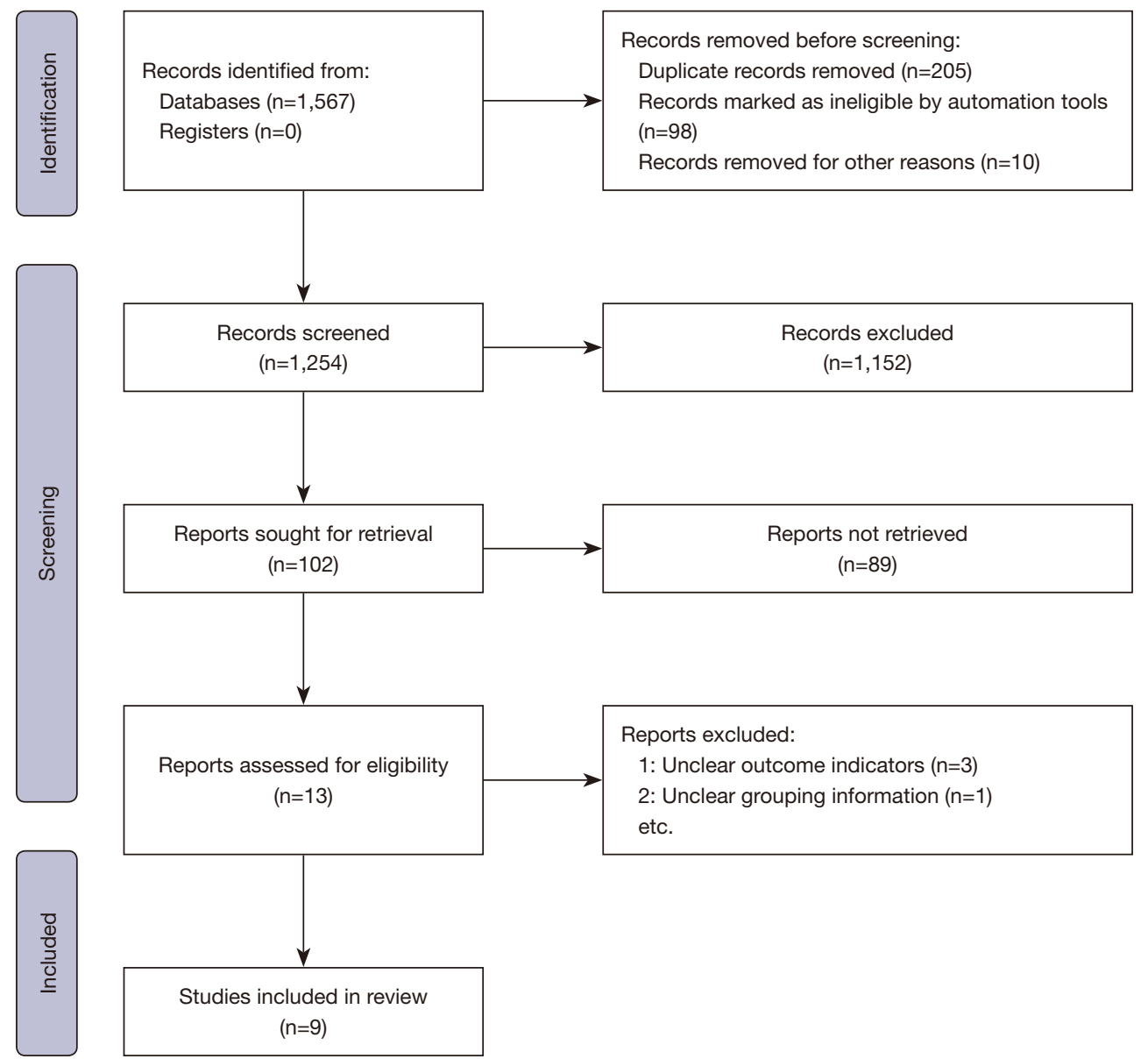

Figure 1 The literature retrieval process.

Table 1 Basic characteristics of the included literatures

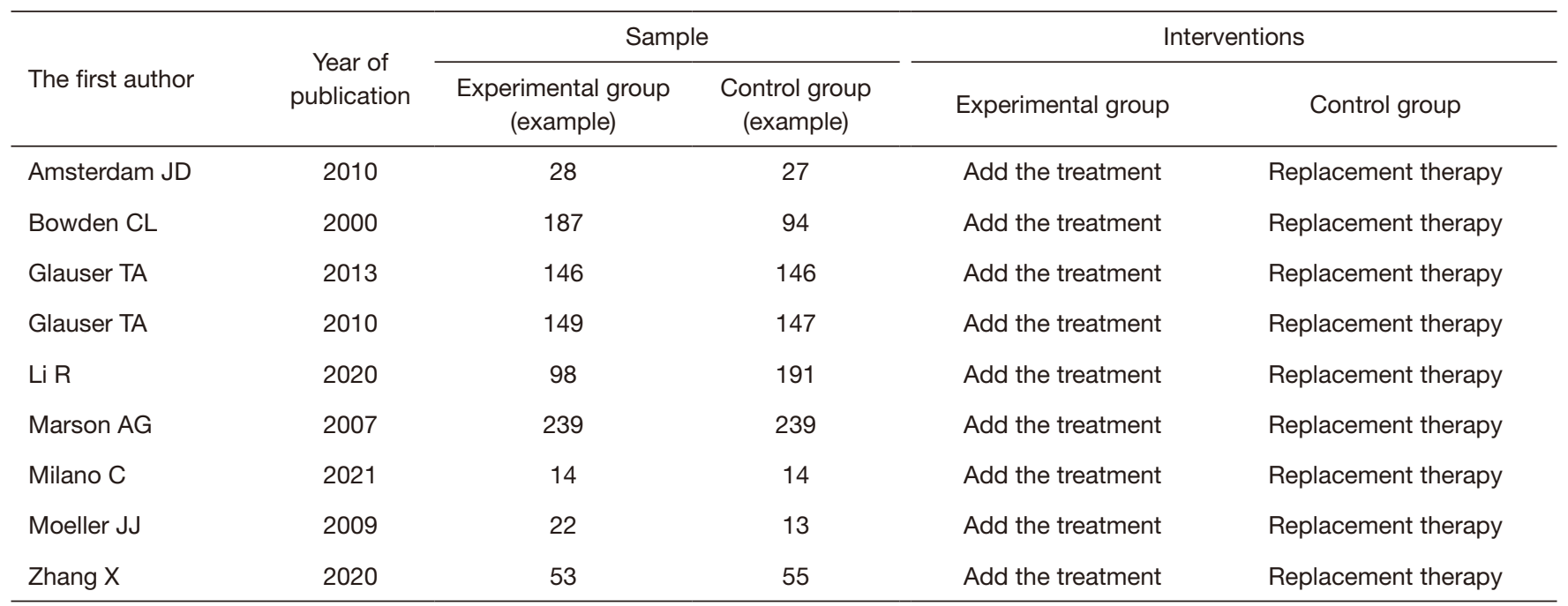




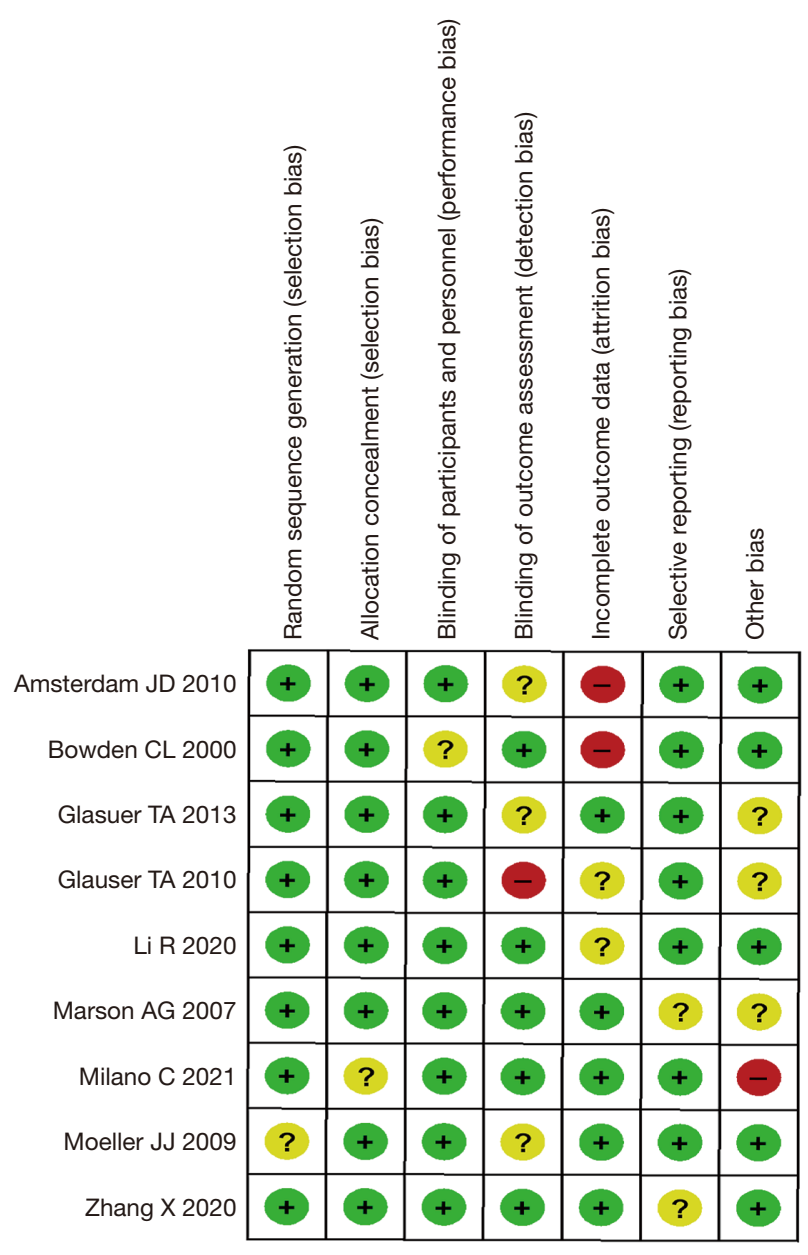

Figure 2 The bias-risk assessment diagram of the included articles. showed that the funnel plot is approximately symmetrical, suggesting that publication bias was not obvious (Figure 7).

\section{Epilepsy improvement rate}

A total of 4 literatures report the epilepsy improvement rate $\left(\mathrm{P}=0.003 ; \mathrm{I}^{2}=79 \%\right)$ and the data were analyzed using the random effects model. Meta-analysis revealed that the epilepsy improvement rate in the experimental group was significantly higher than that in the control group (OR $=4.22 ; 95 \%$ CI: 1.00 to $17.84 ; Z=1.96 ; P=0.05$; Figure 8 ).

The results showed that the funnel plot was approximately symmetrical, suggesting that the publication bias was not obvious (Figure 9).

\section{Discussion}

The mechanisms of action of antiepileptics are very complex and may be related to ion channels and receptors. While there is a paucity of literature on this topic, related studies have shown that the antiepileptic drugs may act on excitatory synapses. For example, phenytoin sodium and other drugs may act on voltage-gated $\mathrm{Na}^{+}$channels. These drugs stabilize the presynaptic membrane and reduce seizures by inhibiting the excitatory synaptic $\mathrm{Na}^{+}$channels, resulting in depolarization and reduced $\mathrm{Ca}^{2+}$ influx (36). Fish antibiotics have also been shown to act on voltagegated $\mathrm{Ca}^{2+}$ channels, and pregabalin and gabapentin can act

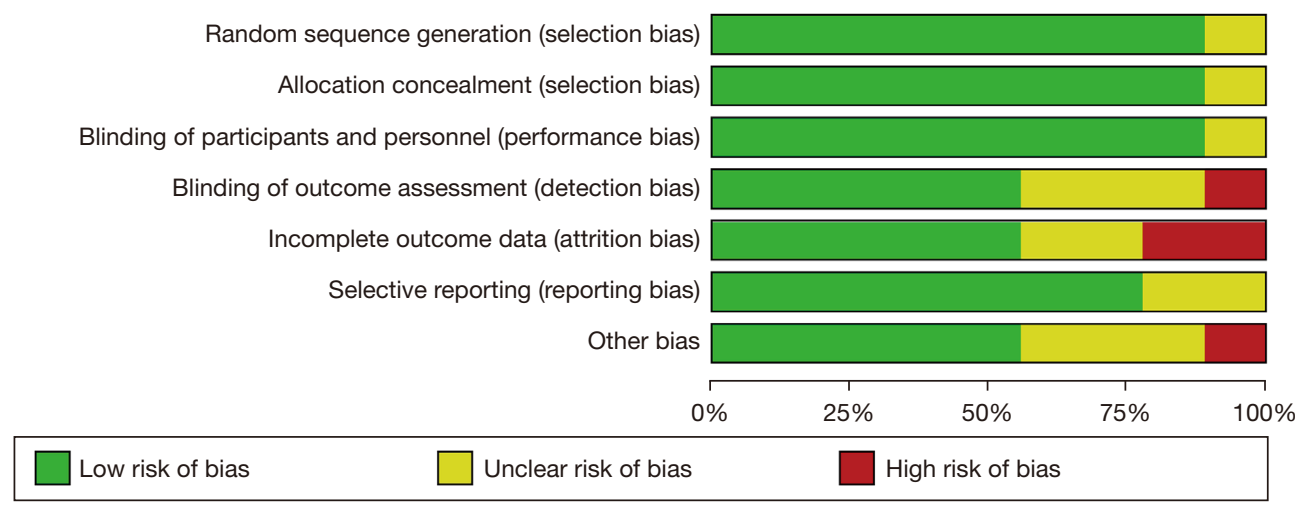

Figure 3 The bias evaluation bar graph of the included articles. 
Table 2 Basic characteristics of the included literatures

\begin{tabular}{|c|c|c|c|c|c|c|}
\hline The first author & Randomization & Binding & $\begin{array}{c}\text { Allocation } \\
\text { concealment }\end{array}$ & $\begin{array}{l}\text { Withdrawals and } \\
\text { dropouts }\end{array}$ & $\begin{array}{l}\text { Reason for dropouts and } \\
\text { withdrawals }\end{array}$ & Jadad \\
\hline Amsterdam JD & Yes & No & NMT & MT & No & 4 \\
\hline Bowden CL & Yes & No & NMT & MT & Yes & 3 \\
\hline Glauser TA & Yes & No & NMT & MT & Yes & 3 \\
\hline Li R & Yes & No & NMT & MT & No & 5 \\
\hline Marson AG & Yes & No & NMT & MT & No & 4 \\
\hline Milano C & Yes & No & NMT & MT & No & 5 \\
\hline Moeller JJ & Yes & No & NMT & MT & Yes & 3 \\
\hline
\end{tabular}

NMT, not mentioned; MT, mentioned.

\begin{tabular}{|c|c|c|c|c|c|c|c|c|c|c|}
\hline Study or Subgroup & $\begin{array}{l}\text { Experim } \\
\text { Events }\end{array}$ & $\begin{array}{l}\text { intal } \\
\text { Total }\end{array}$ & $\begin{array}{l}\text { Contr } \\
\text { Events }\end{array}$ & Total & Weight & $\begin{array}{l}\text { Odds Ratio } \\
\text { M-H, Random, } 95 \% \mathrm{Cl}\end{array}$ & \multicolumn{3}{|c|}{ Odds Ratio } & \\
\hline Amsterdam JD 2010 & 19 & 28 & 13 & 27 & $11.0 \%$ & $2.27[0.76,6.80]$ & & & & \\
\hline Bowden CL 2000 & 116 & 187 & 69 & 94 & $14.3 \%$ & $0.59[0.34,1.02]$ & & & & \\
\hline Glasuer TA 2013 & 118 & 146 & 81 & 146 & $14.4 \%$ & $3.38[2.00,5.72]$ & & & $\varpi$ & \\
\hline Glauser TA 2010 & 86 & 149 & 43 & 147 & $14.6 \%$ & $3.30[2.04,5.34]$ & & & $\neg$ & \\
\hline Li R 2020 & 80 & 98 & 143 & 191 & $13.9 \%$ & $1.49[0.81,2.74]$ & & & & \\
\hline Marson AG 2007 & 227 & 239 & 224 & 239 & $12.9 \%$ & $1.27[0.58,2.77]$ & & & & \\
\hline Milano C 2021 & 12 & 14 & 3 & 14 & $6.5 \%$ & $22.00[3.08,157.34]$ & & & & \\
\hline Moeller JJ 2009 & 22 & 22 & 0 & 13 & $2.3 \%$ & $1215.00[22.76,64865.84]$ & & & & \\
\hline Zhang X 2020 & 47 & 53 & 50 & 55 & $10.1 \%$ & $0.78[0.22,2.74]$ & & & & \\
\hline Total $(95 \% \mathrm{Cl})$ & & 936 & & 926 & $100.0 \%$ & $2.21[1.15,4.27]$ & & & & \\
\hline Total events & 727 & & 626 & & & & & & & \\
\hline Heterogeneity: $\mathrm{Tau}^{2}=$ & $\begin{array}{l}71 ; \mathrm{Chi}^{2} \\
=237(\mathrm{P}\end{array}$ & $\begin{array}{l}47.23 \\
=0.02)\end{array}$ & $d f=8(P$ & $<0.00$ & $01) ; 1^{2}=$ & $83 \%$ & 0.001 & 0.1 & 10 & 1000 \\
\hline
\end{tabular}

Figure 4 A forest plot comparing the total effective rate between the experimental group and the control group. CI, confidence interval.

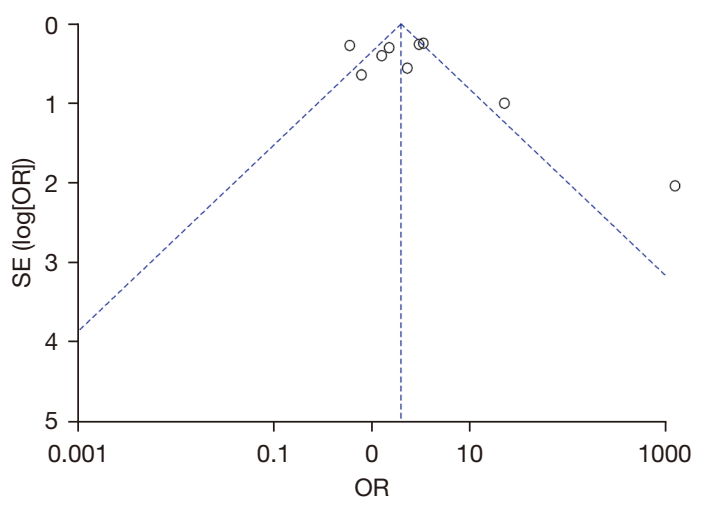

Figure 5 A funnel plot comparing the total effective rate between the experimental group and the control group. SE, standard error; OR, odds ratio. on the $\alpha 2 \delta$ subunit of L-type $\mathrm{Ca}^{2+}$ channels to reduce $\mathrm{Ca}^{2+}$ influx and stabilize the presynaptic membrane by inhibiting $\mathrm{Ca}^{2+}$ channels (37). Gamma-aminobutyric acid (GABA) is a major inhibitory neurotransmitter in the central nervous system that acts on inhibitory synapses and increases the inflow of chloride $\left(\mathrm{Cl}^{-}\right)$(38). GABA concentrations can be increased via two mechanisms. One involves the inhibition of the GABA transporter GAT1 (SLC6A1) which reduces GABA uptake in the presynaptic membrane, thereby increasing GABA concentrations. The second mechanism involves inhibition of the GABA-degrading enzyme GABA transaminase (GABA-T), which reduces GABA degradation and increases $\mathrm{Cl}^{-}$concentrations. Benzodiazepines, barbiturates, and topiramates can all increase the inhibitory 


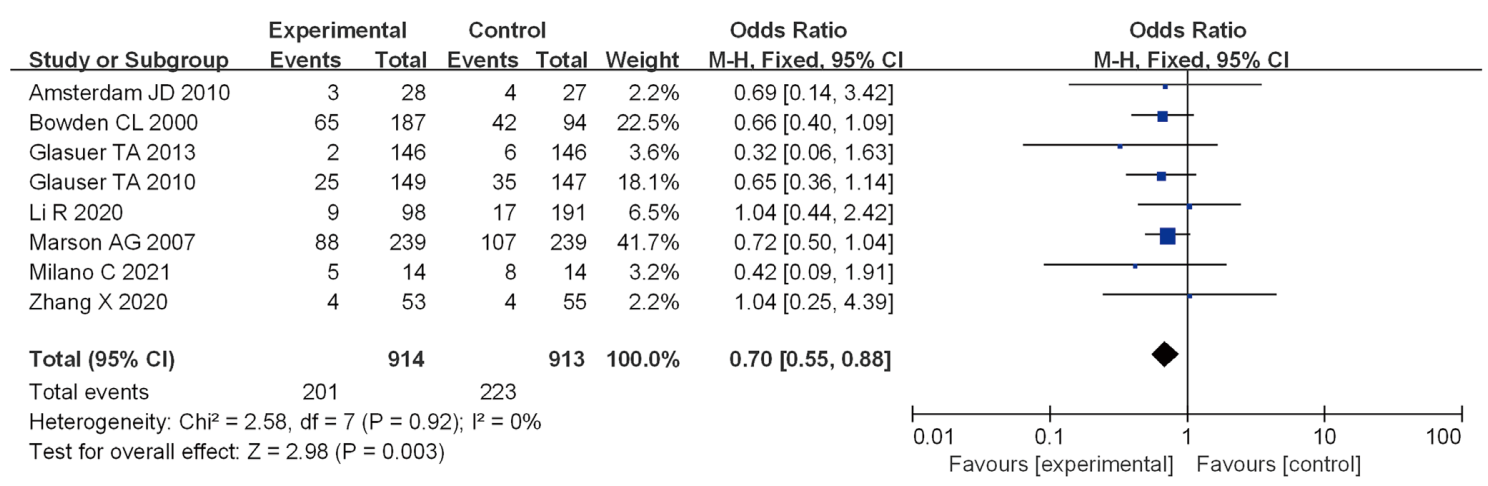

Figure 6 A forest plots comparing the adverse reaction rate in the experimental group and the control group. CI, confidence interval.

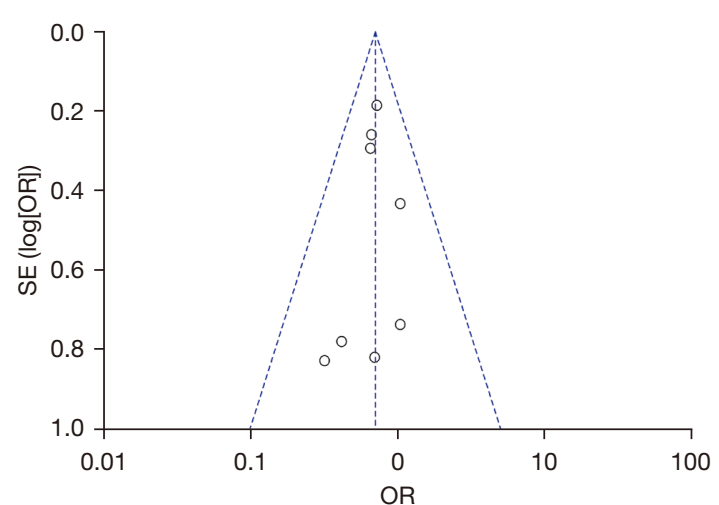

Figure $7 \mathrm{~A}$ funnel plot comparing the adverse reaction rate in the experimental group and the control group. SE, standard error; OR, odds ratio.

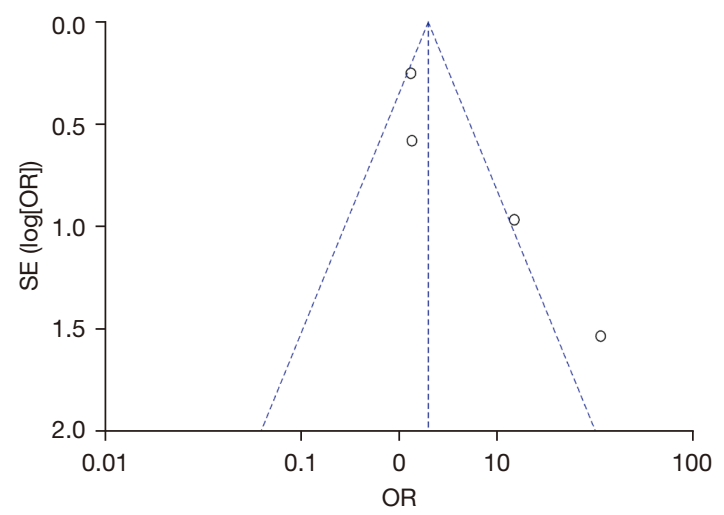

Figure 9 A funnel plot comparing the epilepsy improvement rate in the experimental group and the control group. SE, standard error; OR, odds ratio.

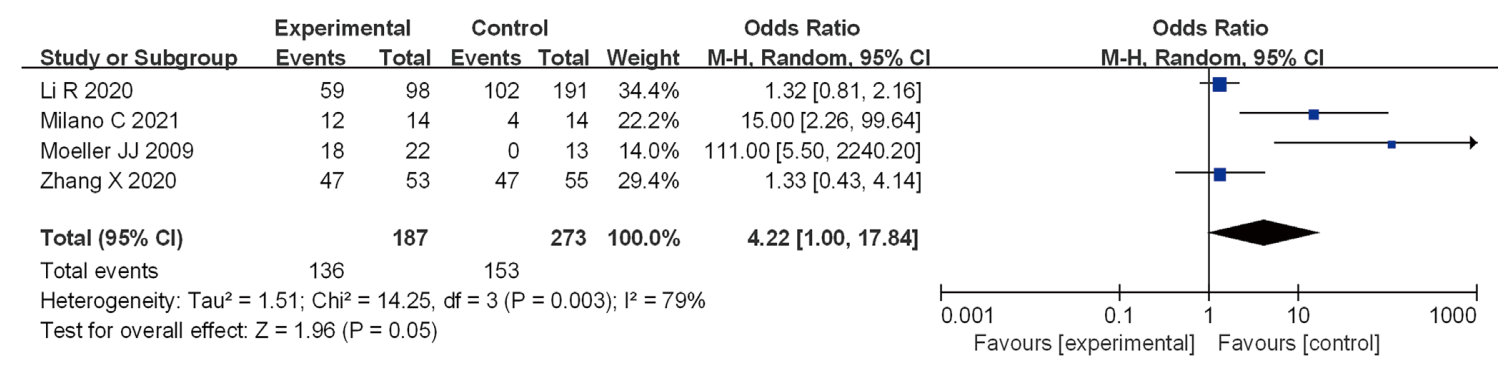

Figure 8 A forest map comparing the epilepsy improvement rate in the experimental group and the control group. CI, confidence interval.

effect of GABA on neuronic hyperexcitation by promoting the opening of $\mathrm{Cl}^{-}$channels $(39,40)$.

Lamotrigine has gradually become the first-choice drug for the treatment of epilepsy. It has the dual effects of preventing and treating epilepsy, with less side effects and low price. Patients who use lamotrigine to treat epilepsy need to monitor liver and kidney function regularly to avoid drug-induced irritation. In short, compared with other studies, lamotrigine has shown excellent efficacy and safety in the treatment of epilepsy. It can be promoted and applied clinically.

In summary, the results of this meta-analysis suggested 
that lamotrigine is superior compared to sodium valproate in the treatment of epilepsy, and furthermore, lamotrigine is effective for patients with epilepsy who are unresponsive to treatment with sodium valproate. Therefore, lamotrigine may have potential therapeutic basis for the clinical treatment of patients with epilepsy. However, future largescale clinical trials of migraine therapy or systematic metaanalyses are warranted to further evaluate the effectiveness of these drugs in epilepsy treatment.

\section{Conclusions}

Starting from the effectiveness of medications for epilepsy, mate analysis was conducted on the effectiveness of lamotrigine in the treatment of epileptic patients who failed to respond to valproate therapy. A total of 9 literatures were included in this meta-analysis. The study demonstrated that good effectiveness could be achieved when sodium valproate was used in the treatment of epileptic patients.

\section{Acknowledgments}

Funding: This study was supported by the National Natural Science Fund (Project No. 81871017).

\section{Footnote}

Reporting Checklist: The authors have completed the PRISMA reporting checklist. Available at https://apm. amegroups.com/article/view/10.21037/apm-21-3555/rc

Conflicts of Interest: All authors have completed the ICMJE uniform disclosure form (available at https://apm. amegroups.com/article/view/10.21037/apm-21-3555/coif). The authors have no conflicts of interest to declare.

Ethical Statement: The authors are accountable for all aspects of the work in ensuring that questions related to the accuracy or integrity of any part of the work are appropriately investigated and resolved.

Open Access Statement: This is an Open Access article distributed in accordance with the Creative Commons Attribution-NonCommercial-NoDerivs 4.0 International License (CC BY-NC-ND 4.0), which permits the noncommercial replication and distribution of the article with the strict proviso that no changes or edits are made and the original work is properly cited (including links to both the formal publication through the relevant DOI and the license). See: https://creativecommons.org/licenses/by-nc-nd/4.0/.

\section{References}

1. Pearce K, Dixon L, D'Arco F, et al. Epilepsy surgery in children: what the radiologist needs to know. Neuroradiology 2020;62:1061-78.

2. Grasso EA, Cacciatore M, Gentile C, et al. Epilepsy in systemic lupus erythematosus. Clin Exp Rheumatol 2021;39:651-9.

3. Pitkänen A, Paananen T, Kyyriäinen J, et al. Biomarkers for posttraumatic epilepsy. Epilepsy Behav 2021;121:107080.

4. Thomas DL, Pierson CR. Neuropathology of Surgically Managed Epilepsy Specimens. Neurosurgery 2020;88:1-14.

5. Golub V, Reddy DS. Cannabidiol Therapy for Refractory Epilepsy and Seizure Disorders. Adv Exp Med Biol 2021;1264:93-110.

6. Wang J, Zhang Y, Zhang H, et al. Nucleus accumbens shell: A potential target for drug-resistant epilepsy with neuropsychiatric disorders. Epilepsy Res 2020;164:106365.

7. Verrotti A, Grasso EA, Cacciatore M, et al. Potential role of brivaracetam in pediatric epilepsy. Acta Neurol Scand 2021;143:19-26.

8. Cross JH. Epilepsy in 2020-a new dawn. Lancet Neurol 2021;20:8-10.

9. Mesraoua B, Deleu D, Hassan AH, et al. Dramatic outcomes in epilepsy: depression, suicide, injuries, and mortality. Curr Med Res Opin 2020;36:1473-80.

10. Gonzalez-Giraldo E, Sullivan JE. Advances in the Treatment of Drug-Resistant Pediatric Epilepsy. Semin Neurol 2020;40:257-62.

11. Bar C, Kuchenbuch M, Barcia G, et al. Developmental and epilepsy spectrum of KCNB1 encephalopathy with longterm outcome. Epilepsia 2020;61:2461-73.

12. Dagar A, Falcone T. Psychiatric Comorbidities in Pediatric Epilepsy. Curr Psychiatry Rep 2020;22:77.

13. Guerri G, Castori M, D'Agruma L, et al. Genetic analysis of genes associated with epilepsy. Acta Biomed 2020;91:e2020005.

14. Gawel K, Langlois M, Martins T, et al. Seizing the moment: Zebrafish epilepsy models. Neurosci Biobehav Rev 2020;116:1-20.

15. Carpio A. The Association between Neurocysticercosis and Epilepsy. Am J Trop Med Hyg 2020;103:1335-6.

16. Mahmood A, Abbasi HN, Ghouri N, et al. Managing 
epilepsy in Ramadan: Guidance for healthcare providers and patients. Epilepsy Behav 2020;111:107117.

17. Steinhoff BJ. Cenobamate-a new perspective for epilepsy treatment. Nervenarzt 2021;92:150-60.

18. Chow CY, Absalom N, Biggs K, et al. Venom-derived modulators of epilepsy-related ion channels. Biochem Pharmacol 2020;181:114043.

19. Verrotti A, Iapadre G, Di Francesco L, et al. Diet in the Treatment of Epilepsy: What We Know So Far. Nutrients 2020;12:2645.

20. Uliel-Sibony S, Chernuha V, Meirson H, et al. Medical treatment of tuberous sclerosis-related epilepsy. Childs Nerv Syst 2020;36:2511-7.

21. Khoo HM, Gotman J, Hall JA, et al. Treatment of Epilepsy Associated with Periventricular Nodular Heterotopia. Curr Neurol Neurosci Rep 2020;20:59.

22. Holmes GL. Drug Treatment of Epilepsy Neuropsychiatric Comorbidities in Children. Paediatr Drugs 2021;23:55-73.

23. Mula M. Pharmacological treatment of focal epilepsy in adults: an evidence based approach. Expert Opin Pharmacother 2021;22:317-23.

24. de Oliveira TVHF, Cukiert A. Deep Brain Stimulation for Treatment of Refractory Epilepsy. Neurol India 2020;68:S268-77.

25. von Wrede R, Helmstaedter C, Surges R. Cannabidiol in the Treatment of Epilepsy. Clin Drug Investig 2021;41:211-20.

26. Upadhya D, Shetty AK. Promise of extracellular vesicles for diagnosis and treatment of epilepsy. Epilepsy Behav 2021;121:106499.

27. Amsterdam JD, Shults J. Efficacy and safety of longterm fluoxetine versus lithium monotherapy of bipolar II disorder: a randomized, double-blind, placebo-substitution study. Am J Psychiatry 2010;167:792-800.

28. Bowden CL, Calabrese JR, McElroy SL, et al. A randomized, placebo-controlled 12-month trial of divalproex and lithium in treatment of outpatients with bipolar I disorder. Divalproex Maintenance Study Group. Arch Gen Psychiatry 2000;57:481-9.

29. Glauser TA, Cnaan A, Shinnar S, et al. Ethosuximide, valproic acid, and lamotrigine in childhood absence epilepsy: initial monotherapy outcomes at 12 months. Epilepsia 2013;54:141-55.

30. Glauser TA, Cnaan A, Shinnar S, et al. Ethosuximide, valproic acid, and lamotrigine in childhood absence epilepsy. N Engl J Med 2010;362:790-9.
31. Li R, Zhou Q, Ou S, et al. Comparison of longterm efficacy, tolerability, and safety of oxcarbazepine, lamotrigine, and levetiracetam in patients with newly diagnosed focal epilepsy: An observational study in the real world. Epilepsy Res 2020;166:106408.

32. Marson AG, Al-Kharusi AM, Alwaidh M, et al. The SANAD study of effectiveness of valproate, lamotrigine, or topiramate for generalised and unclassifiable epilepsy: an unblinded randomised controlled trial. Lancet 2007;369:1016-26.

33. Milano C, Turco F, Pizzanelli C, et al. Response to levetiracetam or lamotrigine in subjects with Juvenile Myoclonic Epilepsy previously treated with valproic acid: A single center retrospective study. Epilepsy Behav 2021;115:107706.

34. Moeller JJ, Rahey SR, Sadler RM. Lamotrigine-valproic acid combination therapy for medically refractory epilepsy. Epilepsia 2009;50:475-9.

35. Zhang X, Zhao W. Comparison of clinical efficacy of oxcarbazepine and lamotrigine combined with escitalopram, and impact on prognostic quality of life in treating patients with epilepsy and depressive disorder. Exp Ther Med 2020;20:146.

36. Holmes M, Flaminio Z, Vardhan M, et al. Cross talk between drug-resistant epilepsy and the gut microbiome. Epilepsia 2020;61:2619-28.

37. Zarnowska IM. Therapeutic Use of the Ketogenic Diet in Refractory Epilepsy: What We Know and What Still Needs to Be Learned. Nutrients 2020;12:2616.

38. Mirzajani S, Ghafouri-Fard S, Habibabadi JM, et al. Altered ANRIL Methylation in Epileptic Patients. J Mol Neurosci 2021;71:193-9.

39. Baldassari S, Musante I, Iacomino M, et al. Brain Organoids as Model Systems for Genetic Neurodevelopmental Disorders. Front Cell Dev Biol 2020;8:590119.

40. Wanleenuwat $P$, Suntharampillai N, Iwanowski P. Antibiotic-induced epileptic seizures: mechanisms of action and clinical considerations. Seizure 2020;81:167-74.

(English Language Editor: J. Teoh)

Cite this article as: $\mathrm{He} \mathrm{J}, \mathrm{Wu} \mathrm{X}$, Zhou D. The efficacy of lamotrigine after failure of the first administration of valproate in treating epilepsy: a systematic review and meta-analysis. Ann Palliat Med 2022;11(1):113-122. doi: 10.21037/apm-21-3555 